\title{
Variáveis fitométricas de macaxeira (Manihot esculenta Crantz) em resposta à aplicação de calagem e gesso agrícola
}

\author{
Ilano Silva Braga do Nascimento \\ Engenheiro Agrônomo formado pela Universidade Federal Rural da Amazônia - UFRA \\ $\bowtie$ p.ilano.nasc@gmail.com \\ Jehmison de Oliveira Barradas \\ Engenheiro Agrônomo formado pela Universidade Federal Rural da Amazônia - UFRA \\ Leonardo Brandão Araújo \\ Engenheiro Agrônomo formado pela Universidade Federal Rural da Amazônia - UFRA \\ Mauro Junior Borges Pacheco \\ Graduando do curso de Agronomia pela Universidade Federal Rural da Amazônia - UFRA \\ Thiago Costa Viana \\ Graduando do curso de Agronomia pela Universidade Federal Rural da Amazônia - UFRA \\ Andreza Mayra Baena Souza de Jesus \\ Graduanda do curso de Agronomia pela Universidade Federal Rural da Amazônia - UFRA \\ Jessivaldo Rodrigues Galvão \\ Professor Doutor (Agronomia) da Universidade Federal Rural da Amazônia - UFRA
}

Recebido em 12 de setembro de 2019

Aceito em 8 de outubro de 2019

\begin{abstract}
Resumo:
A ONU elegeu a mandioca como o alimento do século XXI. Apresenta a possibilidade de aproveitamento integral, a folha é aproveitada para alimentação animal e humana, enquanto as manivas podem ser utilizadas para propagação ou silagem. No Brasil a mandioca é cultivada com o mínimo uso de insumos ou nenhum, em sistema de derruba-e-queima, embora apresentando tolerância a acidez e escassez de recursos, o cultivo contínuo, sem reposição dos nutrientes exportados, pode ocasionar o esgotamento das reservas nutricionais e levar a degradação. O objetivo foi avaliar a fitometria das variáveis de Manihot esculenta Crantz, quando submetidas à aplicação de calcário e gesso. O experimento foi realizado em campo, em 2018, na área experimental do Departamento de Solos, pertencente ao Instituto de Ciências Agrárias da Universidade Federal Rural da Amazônia - UFRA, localizada no município de Belém, Pará. $O$ delineamento experimental foi em blocos casualizados, em arranjo fatorial $2 \times 3$, com seis repetições, totalizando 36 unidades experimentais. Os fatores foram: 2 variedades de macaxeira (Caeté e Manteiguinha) e 3 correções (calcário, gesso, calcário + gesso). Os tratamentos consistiram da correção do solo com calcário (T1), gesso (T2), e a combinação de ambos (T3), os quais se implantaram as variedades Caeté (C1) e Manteiguinha (C2). As variáveis fitométricas foram: altura da planta (AP), altura da primeira ramificação (APR), matéria fresca das folhas (MFF), matéria seca das folhas (MSF), matéria fresca das estacas (MFE) e índice de colheita (IC). As aplicações isoladas e combinadas de calcário e gesso promoveram resultados significativos para as variáveis analisadas.
\end{abstract}

Palavras-chave: Mandioca, correção do solo, gessagem, fitometria. 


\section{Phytometric variables of manioc (Manihot esculenta Crantz) in response to liming and plaster application}

Abstract:

The UN has elected cassava as the food of the 21st century. It has the possibility of full utilization, the leaf is used for animal and human feed, while the handles can be used for propagation or silage. In Brazil, cassava is cultivated with minimal or no use of inputs in a knock down-and-burn system, although with tolerance to acidity and scarcity of resources, continuous cultivation without replacement of exported nutrients may cause depletion of reserves. nutritional and lead to degradation. The objective was to evaluate the phytometry of the Manihot esculenta Crantz variables, when submitted to limestone and plaster application. The experiment was carried out in the field, in 2018, in the experimental area of the Department of Soils, belonging to the Institute of Agrarian Sciences of the Universidade Federal Rural da Amazonia UFRA, located in the city of Belém, Pará. The experimental design was a randomized block design. $2 \times 3$ factorial arrangement, with six replications, totaling 36 experimental units. The factors were: 2 varieties of macaxeira (Caeté and Manteiguinha) and 3 corrections (limestone, plaster, limestone + plaster). The treatments consisted of soil correction with limestone (T1), plaster (T2), and the combination of both (T3), which were implanted the varieties Caeté (C1) and Manteiguinha (C2). The phytometric variables were: plant height (AP), first branch height (APR), leaf fresh matter (MFF), leaf dry matter (MSF), cuttings fresh matter (MFE) and harvest index (CI). . The isolated and combined applications of limestone and plaster promoted significant results for the analyzed variables.

Keywords: Cassava, soil correction, plaster, phytometry.

\section{Variables fitométricas de la mandioca (Manihot esculenta Crantz) en respuesta a la aplicación de cal y yesoítulo}

\section{Resumen:}

La ONU ha elegido la yuca como alimento del siglo XXI. Presenta la posibilidad de una utilización completa, la hoja se usa para alimentación animal y humana, mientras que la mantis se puede usar para propagación o ensilaje. En Brasil, la yuca se cultiva con un uso mínimo o nulo de insumos, en un sistema de desmontaje, aunque con tolerancia a la acidez y escasez de recursos. nutricional y conducir a la degradación. El objetivo fue evaluar la fitometría de las variables de Manihot esculenta Crantz, cuando se sometió a aplicación de caliza y yeso. El experimento se realizó en el campo, en 2018, en el área experimental del Departamento de Suelos, perteneciente al Instituto de Ciencias Agrarias de la Universidad Federal Rural de Amazonia - UFRA, ubicada en la ciudad de Belém, Pará. Arreglo factorial 2x3, con seis repeticiones, totalizando 36 unidades experimentales. Los factores fueron: 2 variedades de macaxeira (Caeté y Manteiguinha) y 3 correcciones (caliza, yeso, caliza + yeso). Los tratamientos consistieron en la corrección del suelo con piedra caliza (T1), yeso (T2) y la combinación de ambos (T3), en los que se implantaron las variedades Caeté (C1) y Manteiguinha (C2). Las variables fitométricas fueron: altura de la planta (AP), altura de la primera rama (APR), materia fresca de la hoja (MFF), materia seca de la hoja (MSF), esquejes de materia fresca (MFE) e índice de cosecha (IC). Las aplicaciones aisladas y combinadas de piedra caliza y yeso promovieron resultados significativos para las variables analizadas.

Palabras clave: Yuca, corrección de suelos, yeso, fitometría. 


\section{INTRODUÇÃO}

A Organização das Nações Unidas (ONU) elegeu a mandioca como o alimento do século XXI (EMBRAPA, 2017). O Brasil alcançou a $5^{\text {a }}$ posição de país com a maior quantidade produzida da cultura, foram 18.876.470 toneladas (FAO, 2018).

A macaxeira se diferencia da mandioca brava por apresentar baixos teores de ácido cianídrico (HCN) na polpa crua de raízes frescas, geralmente abaixo de $50 \mathrm{mg} / \mathrm{kg}$ de polpa (DIAS et al., 2003). A macaxeira utilizada em consumo fresco, processadas, ou na indústria, para consumo humano cozida, frita, massa para bolos, purês, suflês e outras variedades (PONTE, 2008). Mas, apresenta a possibilidade de aproveitamento integral da planta, a folha é aproveitada para alimentação animal e humana, enquanto as manivas podem ser utilizadas para propagação ou silagem (CARDOSO e SOUZA, 2002). Segundo Souza (2007 apud SANTOS et al., 2009), as folhas da mandioca poderiam agregar valor na renda dos produtores, na forma de alimento animal ou para humanos. Carvalho e Kato (1987) indicam uma produção de aproximadamente 9 t.ha ${ }^{-1}$ de matéria fresca da parte aérea da mandioca, considerando a densidade de 20.000 plantas/ha. Para Modesto et al. (1999) se fosse aproveitado o refugo da parte aérea da mandioca, atingiria-se a produção de 14,3 milhões de toneladas de matéria fresca disponível para a alimentação animal.

Na região Amazônica a macaxeira é cultivada com quase nenhum insumo (SOUZA et al., 2009), em geral, sob sistema de derruba-e-queima (CRAVO et al., 2005), embora apresente tolerância à acidez e escassez de recursos, sob cultivo contínuo sem a reposição dos nutrientes exportados, pode ocorrer o esgotamento dos nutrientes e a degradação (MIRANDA et al., 2005).

O objetivo do trabalho foi avaliar as variáveis fitométricas de Manihot esculenta Crantz, quando submetidas à aplicação de calcário e gesso.

\section{METODOLOGIA}

O experimento foi realizado em campo, no período de 30/03 até 19/11 de 2018 na área experimental do Departamento de Ciências do Solo - ICA - UFRA, Belém-PA. Segundo a classificação de Köppen, o clima de Belém é caracterizado como Equatorial Afi, (KOTTEK, et 
al., 2006). No período da realização do experimento as precipitações pluviométricas registradas pelas estações automáticas de Belém-PA totalizaram de 2.104,57 mm, segundo o Sistema de Suporte à Decisão na Agropecuária do Instituto Nacional de Meteorologia (SISDAGRO). O solo da área foi classificado como Latossolo Amarelo Distrófico típico, de textura média (EMBRAPA, 2006). Para manejo nutricional da cultura, o solo foi coletado sob profundidade de $0-20 \mathrm{~cm}$ e submetido a análise química seguindo metodologia descrita em Embrapa (1997). O resultado da análise química do solo consta na Tabela 1.

Tabela 1 - Caracterização química do Latossolo Amarelo, amostrado na camada de 0$20 \mathrm{~cm}$ de profundidade. Belém, PA, 2016.

\begin{tabular}{cccccccccccc}
\hline AMOSTRA & $\mathbf{p H}$ & $\mathbf{N}$ & $\begin{array}{c}\mathbf{P} \\
(1)\end{array}$ & $\begin{array}{c}\mathrm{K}^{+} \\
(1)\end{array}$ & $\underset{(1)}{\mathrm{Na}^{+}}$ & $\mathrm{Ca}^{2+(2)}$ & $\underset{(2)}{\mathrm{Mg}^{2+}}$ & $\mathrm{Al}^{3+(3)}$ & $\begin{array}{c}\mathrm{H}+\mathrm{Al} \\
(3)\end{array}$ & $\mathbf{m}$ & $\mathbf{V}$ \\
\hline Profundidade & $\mathrm{H}_{2} \mathrm{O}$ & $\%$ & \multicolumn{2}{c}{$\mathrm{mg} \mathrm{dm}^{-3}$} & & $\mathrm{Cmol}_{\mathrm{c}} \mathrm{dm}^{-3}$ & & \multicolumn{2}{c}{$\%$} \\
$0-20$ & 4,6 & 0,05 & 6 & 22 & 14 & 0,3 & 0,2 & 1,4 & 6,23 & 71,57 & 8,19 \\
\hline
\end{tabular}

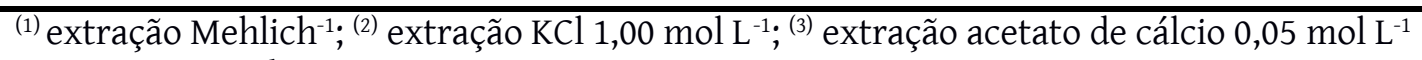
Fonte: Acervo dos autores.

O delineamento foi em blocos casualizados, em arranjo fatorial $2 \times 3$, com seis repetições, totalizando 36 unidades experimentais. Os fatores foram: 2 variedades de macaxeira (Caeté e Manteiguinha) e 3 correções (calcário, gesso, calcário + gesso). Os tratamentos foram: aplicação de calcário (T1), aplicação de gesso (T2), e a combinação de calcário+gesso (T3), variedades Caeté (C1) e Manteiguinha (C2). A correção do solo com calcário ocorreu de acordo com a recomendação, em função da análise de solo, sob a metodologia da neutralização do alumínio trocável (EMBRAPA, 2016). A recomendação de calagem foi de 1,34 t.ha-1 PRNT 91\% (67 mg de calcário dolomíticos por planta, densidade de 20.000 plantas.ha $^{-1}$ ) e gessagem 1,2 t.ha ${ }^{-1}$ (60 mg por planta de gesso agrícola). A correção do solo em subsuperfície com o gesso agrícola seguiu a recomendação de acordo com a classificação textural do solo para culturas anuais (SBCS, 2007). As parcelas experimentais foram espaçadas na proporção $1 \times 1 \times 0,5 \mathrm{~m}$. A área total foi de $8 \times 11 \mathrm{~m}, 88 \mathrm{~m}^{2}$.

Antes do preparo das linhas de plantio foram abertas 5 covas, para a aplicação dos tratamentos. Após a aplicação dos corretivos, realizou-se a construção de camalhões com enxada. 20 dias decorridos após a aplicação dos tratamentos, aplicou-se a fonte fosfórica e em seguida foram plantadas as manivas sementes, de forma manual, da variedade Caeté e da variedade Manteiguinha. Um dia após o plantio, foi aplicado o herbicida pré-emergente 
Flumyzin $^{\circledast}$, concentração de Flumioxazina de 500 g.kg-1 , sob a dosagem de 200 g.ha-1 ${ }^{-1} 45$ dias após o plantio foi realizada a adubação de cobertura, seguindo a recomendação de acordo com a análise de solo. A adubação ocorreu seguindo a recomendação para o Estado do Pará, (EMBRAPA, 2007). Utilizou-se como fonte nitrogenada, fosfórica, potássica e de micronutrientes: uréia, superfosfato triplo (SFT), cloreto de potássio (KCl), e FTE-BR12, respectivamente. $\mathrm{O}$ controle de plantas daninhas foi realizado com aplicação de herbicidas e controle mecânico de acordo com a necessidade. Inspeções fitossanitárias eram realizadas semanalmente.

O experimento foi conduzido por 235 dias. Foram coletadas as 3 plantas centrais de cada tratamento, na linha de plantio. As variáveis de resposta foram: altura da planta (AP), altura da primeira ramificação (APR), matéria fresca das folhas (MFF), matéria seca das folhas (MSF), matéria fresca das estacas (MFE) e índice de colheita (IC). A AP, APR foram mensuradas em campo, com auxílio de fita métrica, conforme proposto por Fukuda e Guevara (1998). A MFF e MFE foram mensuradas com auxílio de balança digital, também no campo. A MSF foi avaliada segundo a metodologia de Detmann et al. (2012). O IC foi determinado pela relação dos valores médios de MFR/Matéria Fresca Total, conforme Fukuda e Guevara (1998).

Os resultados do experimento foram submetidos à análise de variância, comparadas pelo teste de Tukey a $5 \%$ de probabilidade. Para a obtenção dos resultados utilizou-se o programa estatístico Sisvar 5.3 (FERREIRA, 2011).

\section{RESULTADOS}

As variáveis AP, MFF, MSF, MFE, NR e MFR foram influenciadas significativamente pela aplicação de calcário e gesso, isoladamente, e em suas interações. CR e DR não foram influenciados apenas com a correção. A variável MFR apresentou diferenças apenas com as variedades, já o IC foi influenciado pelas variedades e a interação entre os tratamentos. A APR não apresentou resultados significativos para os tratamentos e suas interações (Tabela 2). 
Tabela 2 - Resumo da análise de variância para as variáveis AP, APR, MFF, MSF, MFE e IC da macaxeira (Manihot esculenta Crantz) sob efeito da aplicação de calcário e gesso agrícola.

\begin{tabular}{lccccccc}
\hline FONTE DE VARIAÇÃo & GL & AP & APR & MFF & MSF & MFE & IC \\
\hline Correção & 2 & $*$ & Ns & $* *$ & $* *$ & $* *$ & ns \\
Variedade & 1 & $*$ & Ns & $* *$ & $* *$ & $* *$ & $* *$ \\
Cor x Var & 2 & $* *$ & Ns & $* *$ & $* *$ & $* *$ & $*$ \\
\hline Erro & 25 & - & - & - & - & - & - \\
\hline CV \% & - & 6,85 & 41,57 & 4,2 & 4,2 & 5,63 & 8,77 \\
\hline
\end{tabular}

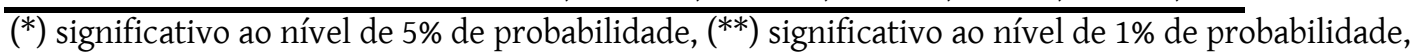
(ns) não significativo ao nível de $5 \%$ de probabilidade.

Fonte: Acervo dos autores.

\section{Altura da planta (AP) e altura da primeira ramificação (APR)}

A interação das aplicações de calcário e gesso com as variedades, demonstram diferenças entre os tratamentos. Observou-se melhores resultados na variedade Caeté com a aplicação do calcário, fato não ocorrido com a Manteiguinha que apresentou resultados semelhantes com as correções aplicadas. Apesar de apresentar diferenças entre as variedades com a relação a altura das plantas, a altura da primeira ramificação não foi influenciada pelos tratamentos (Tabela 3).

Tabela 3 - Variável Altura da planta (AP) e altura da primeira ramificação (APR) em função dos tratamentos.

\begin{tabular}{lccl}
\hline \multicolumn{4}{c}{ AP $(\mathbf{m})$} \\
\hline Variedades & Calcário & Gesso & Cal+Ges \\
Caeté & $2,07 \mathrm{Aa}$ & $1,73 \mathrm{Ba}$ & $1,85 \mathrm{Ba}$ \\
Manteiguinha & $1,75 \mathrm{Ab}$ & $1,76 \mathrm{Aa}$ & $1,86 \mathrm{Aa}$ \\
\hline \multicolumn{5}{c}{ APR $(\mathbf{m})$} \\
\hline \multicolumn{5}{c}{6,85} \\
\hline Variedades & Calcário & $\mathrm{Gesso}$ & $\mathrm{Cal+Ges}$ \\
Caeté & $1,04 \mathrm{Aa}$ & $0,83 \mathrm{Aa}$ & $0,85 \mathrm{Aa}$ \\
Manteiguinha & $1,05 \mathrm{Aa}$ & $1,19 \mathrm{Aa}$ & $0,87 \mathrm{Aa}$ \\
\hline \multicolumn{5}{c}{41,57} \\
\hline
\end{tabular}

Letras maiúsculas na horizontal comparam as variedades entre os tratamentos. Letras minúsculas na vertical comparam as variedades em cada tratamento.

Fonte: Acervo dos autores. 


\section{Matéria fresca das folhas (MFF), matéria seca das folhas (MSF)}

Com base nos dados, foram verificadas diferenças significativas entre os tratamentos para as variáveis MFF e MSF. A aplicação de gesso, isoladamente, apresentou melhores produtividade de MFF e MSF entre os tratamentos aplicados. Os menores resultados de MFF e MSF foram encontrados sob a aplicação combinada de calcário + gesso para a variedade Manteiguinha (Tabela 4).

Tabela 4 - Matéria fresca das folhas (MFF) e Matéria seca das folhas (MSF) em função dos tratamentos.

\begin{tabular}{lccc}
\hline \multicolumn{4}{c}{ MFF (g) } \\
\hline Variedades & Calcário & Gesso & Cal+Ges \\
Caeté & $147,23 \mathrm{Ba}$ & $204,78 \mathrm{Aa}$ & $126 \mathrm{Ca}$ \\
Manteiguinha & $52,07 \mathrm{Bb}$ & $62,20 \mathrm{Ab}$ & $45,42 \mathrm{Cb}$ \\
\hline CV\% & 4,2 \\
\hline \multicolumn{4}{c}{ MSF (g) } \\
\hline Variedades & Calcário & Gesso & Cal+Ges \\
Caeté & $70,67 \mathrm{Ba}$ & $98,29 \mathrm{Aa}$ & $60,48 \mathrm{Ca}$ \\
Manteiguinha & $24,99 \mathrm{Bb}$ & $29,86 \mathrm{Ab}$ & $21,80 \mathrm{Cb}$ \\
\hline CV\% & 4,2 & \\
\hline
\end{tabular}

Letras maiúsculas na horizontal comparam as variedades entre os tratamentos. Letras minúsculas na vertical comparam as variedades em cada tratamento.

Fonte: Acervo dos autores.

\section{Matéria fresca das estacas (MFE)}

Os resultados encontrados com a produção de massa fresca das estacas (MFE), se assemelham aos da MFF e MSF, ou seja, variedade Caeté sob aplicação de gesso expressou melhores resultados, para Manteiguinha a maior produção ocorreu na interação do calcário + gesso (Tabela 5). 
Tabela 5 - Matéria fresca das estacas (MFE) em função dos tratamentos.

\begin{tabular}{lccc}
\hline \multicolumn{4}{c}{ MFE (g) } \\
\hline Variedades & Calcário & Gesso & Cal+Ges \\
Caeté & $516,68 \mathrm{Ba}$ & $602,78 \mathrm{Aa}$ & $441,60 \mathrm{Ca}$ \\
Manteiguinha & $218,36 \mathrm{Bb}$ & $177,68 \mathrm{Cb}$ & $409,41 \mathrm{Ab}$ \\
\hline CV\% & 5,63 & & \\
\hline
\end{tabular}

Letras maiúsculas na horizontal comparam as variedades entre os tratamentos. Letras minúsculas na vertical comparam as variedades em cada tratamento.

Fonte: Acervo dos autores.

\section{Índice de colheita (IC)}

Comparando os tratamentos com calcário, gesso e suas combinações, verificou-se haver apenas diferenças numéricas de IC entre as variedades, não havendo diferenças estatísticas. (Tabela 6).

Tabela 6 - Índice de colheita (IC) em função dos tratamentos.

\begin{tabular}{llcc}
\hline \multicolumn{3}{c}{ IC (\%) } \\
\hline Variedade & Calcário & Gesso & Cal+Ges \\
Caeté & $64,01 \mathrm{Ab}$ & $63,65 \mathrm{Ab}$ & $70,13 \mathrm{Aa}$ \\
Manteiguinha & $74,80 \mathrm{Aa}$ & $79,85 \mathrm{Aa}$ & $72,09 \mathrm{Aa}$ \\
\hline CV\% & 8,77 & & \\
\hline
\end{tabular}

Letras maiúsculas na horizontal comparam as variedades entre os tratamentos. Letras minúsculas na vertical comparam as variedades em cada tratamento.

Fonte: Acervo dos autores.

\section{DISCUSSÃO}

\section{Altura da planta (AP) e altura da primeira ramificação (APR)}

Avaliando os resultados encontrados na pesquisa, verificamos que a variedade Caeté, sob efeito da correção do solo com calcário, apresentou maior porte, fato que pode estar ligado às suas características genéticas e disponibilidade hídrica no período vegetativo da cultura, durante a execução do experimento, haja visto que em ambas as variedades, as manivas sementes selecionadas para a implantação do experimento foram colhidas aos 6 meses e plantadas após uma semana do corte. 
Para Valle (1990), estas variáveis estão relacionadas à maior facilidade do manejo e aplicação dos tratos culturais, ou seja, quanto maior a altura das plantas e maior a altura da primeira ramificação, maior será a facilidade de manejo da cultura. Valle (1990) também defende que existe correlação da AP e APR com a produtividade, embora em menor nível quando comparado à matéria verde da parte aérea. Baseado nas informações dos autores, e relacionando com os resultados do experimento, identificamos que a variedade Caeté seria a mais promissora nessa avaliação.

As variações de altura da planta também podem estar relacionadas aos componentes genéticos e associados à influência do ambiente (RIMOLDI et al., 2006). Tezara et al. (2002) afirmam em seu experimento que o crescimento das plantas, sofreu grande influência da disponibilidade hídrica. No caso da macaxeira o período mais sensível é dos 15 aos 90 dias após o plantio, onde ocorre a formação do sistema radicular, o qual influenciará no desenvolvimento da parte aérea da planta.

Oliveira et al. (2006) advertem que a altura da planta é variável e depende do tipo de ramificação. Coqueiro (2013) defende que a diferença da altura da primeira ramificação se deve a fatores predominantemente genéticos. O porte da variedade Caeté se demonstrou ser do tipo aberto, com hábito de ramificação tricotômico, enquanto que a variedade Manteiguinha apresentou porte do tipo cilíndrico e hábito de ramificação ereto (ANDRADE et al., 2011). Comparados ao estudo realizado, estes fatos podem ter influenciado na APR, com seus resultados não significativos.

\section{Matéria fresca das folhas (MFF), matéria seca das folhas (MSF) e matéria fresca das estacas (MFE)}

No experimento, a MFF da variedade Caeté sob efeito da gessagem alcançou a produção de 4 t.ha ${ }^{-1}$, possivelmente influenciado pelo efeito do gesso agrícola na correção em profundidade, disponibilizando maior quantidade de nutrientes ao longo do perfil do solo. Apesar dos rendimentos terem se apresentado menores em relação aos encontrados por Carvalho e Kato (1987), o efeito da gessagem é um fator que pode favorecer maior estruturação do solo, provocando o alongamento de raízes e maior absorção de nutrientes.

Em estudos realizados com 4 variedades no município de Presidente Tancredo NevesBA, com clima quente e úmido, o rendimento de matéria seca das variedades estudadas 
estaciona em torno de 25\% (EMBRAPA, 2007). Em contrapartida, no experimento com as variedades Caeté e Mateiguinha, o percentual médio de matéria seca das folhas chegou a 48\% em função dos tratamentos aplicados. É possível que as variedades apresentem características genéticas para acúmulo da matéria seca das folhas. Com isso, acreditamos que há necessidade de maiores estudos com relação às variedades testadas no experimento, pois apresentam um bom potencial produtivo.

A possibilidade do uso das folhas como fonte de proteínas de baixo custo na alimentação animal e humana foi estudada por Carvalho (1983) e Ferreira et al. (2008). Segundo Souza (2007 apud SANTOS et al., 2009), este produto poderia agregar valor na renda dos produtores, na forma de alimento animal ou para humanos. Carvalho e Kato (1987) indicam uma produção de aproximadamente 9 t.ha ${ }^{-1}$ de matéria fresca da parte aérea da mandioca, considerando a densidade de 20.000 plantas por hectare. Para Modesto et al. (1999) se fosse aproveitado o refugo da parte aérea da mandioca, atingiria-se a produção de 14,3 milhões de toneladas de matéria fresca disponível para a alimentação animal.

Carvalho (1994) descreve que, apenas cerca de 20 \% do total de ramas produzidas numa área são aproveitadas para o replantio. É possível que acúmulo da matéria fresca nas estacas das variedades avaliadas em função dos tratamentos pode colaborar para a qualidade do material final a ser propagado, contribuindo para maior aproveitamento do percentual de ramas produzidas na lavoura. Os tratamentos estudados podem ter influenciado na maior concentração de MFE, fato este que pode predispor em características de melhor relação medula x cortéx de estaca e, consequentemente, maior qualidade de brotação, precocidade e desenvolvimento das variedades (LOZANO et al., 1977; CONCEIÇÃO, 1983; BEZERRA, 2012).

\section{Índice de colheita (IC)}

É provável que estes resultados tenham ocorrido em função do porte das plantas associado às características genéticas expressadas ao final dos 7 meses, período em que houve perda considerável da parte aérea de ambas as variedades.

Valle et al. (2005) relataram que um bom índice de colheita está acima de 50\%. Maiores valores de IC estão relacionados a maiores produtividades, devido ser uma característica de genótipos que alocam maior biomassa às raízes, isto permite maior densidade populacional pela redução proporcional da parte aérea. Lenis et al. (2006) defendem que variedades que apresentam maiores índices de colheita também demonstram maiores valores de retenção 
foliar. O mecanismo é explicado devido a planta reter por mais tempo folhas dotadas de eficiência no uso de água, há tendência de ocorrer maior acúmulo de fotossintatos nas raízes tuberosas, fazendo com que haja aumento no IC.

\section{CONSIDERAÇÕES FINAIS}

A variedade Caeté apresenta melhores resultados com a aplicação de calcário e gesso.

A aplicação de gesso influencia em maiores resultados de matéria fresca e seca de folhas para as variedades.

O calcário e o gesso agrícola, aplicados isoladamente, além da combinação entre eles, elevam a média do índice de colheita da macaxeira.

\section{REFERÊNCIAS}

ANDRADE, A. C. B.; VIANA, A. E. S.; CARDOSO, A. D.; SANTOS, V. S.; LOPE, S. C.; FOGAÇA, J. J. N. L. CARACTERIZAÇÃO DE VARIEDADES DE MANDIOCA DE MESA POR MEIO DE DESCRITORES MORFOLÓGICOS. Universidade Estadual do Sudoeste da Bahia/UESB, Vitória da Conquista - BA, 2011.

BEZERRA, V.S. Maniva-semente: como selecionar e conservar. Macapá: Embrapa Amapá. 5p. (Comunicado Técnico 125). $2012 . \quad$ Disponível em: http://ainfo.cnptia.embrapa.br/digital/bitstream/item/101452/1/Comunicado-Tecnico-125-manivasemente.pdf. Acesso em: 15/02/2019.

CARDOSO, C. E. L.; SOUZA, J. S. Importância, potencialidades e perspectivas do cultivo de mandioca na América Latina. In: CEREDA, M. P. (Coord.) Agricultura: tuberosas amiláceas Latino Americanas. São Paulo: Fundação Cargill, p. 29-47, 2002. (Série Culturas de Tuberosas Amiláceas Latino Americanas, 2).

CARVALHO, J. L. H. 1983. A mandioca, raiz e parte aérea na alimentação animal. EMBRAPA.

CARVALHO, J. L. H. A mandioca: raiz e parte aérea na alimentação animal. Campinas: CATI, 1994. 9 p.

CARVALHO, V.D.; KATO, M. S. Potencial de utilização da parte aérea da mandioca. Informe Agropecuário, Belo Horizonte, v. 13, n. 145, p. 23-28, 1987.

CONCEIÇÃO, A. J. A mandioca. São Paulo: Nobel, 1983. 382 p.

COQUEIRO, G. R. AVALIAÇÃo DE GENÓTIPOS DE MANDIOCA AMARELA (Manihot esculenta Crantz) PARA PRODUÇÃO E QUALIDADE NUTRICIONAL DA RAIZ NAS CONDIÇÕES EDAFOCLIMÁTICAS DO NORDESTE DO ESTADO DO PARÁ. 2013. 42 f. Tese (Doutorado em Agronomia). Faculdade de Ciências Agronômicas da Universidade Estadual Paulista "JÚLIO DE MESQUITA FILHO", Botucatu, 2013.

CRAVO, M. S.; SMYTH, T. J. Atributos físico-químicos e limitações dos solos de áreas produtoras de feijão-caupi no Nordeste do Estado do Pará. In: CONGRESSO BRASILEIRO CIÊNCIA DO SOLO, 30., 2005, Recife. Anais... Recife: SBCS, 2005. 1 CD-ROM. 
DETMANN, E. [et al.] (ed.). Métodos para análise de alimentos. Visconde de Rio Branco, MG: Suprema. 2012. 214 p. ISBN 978-858-17-9020-6.

DIAS, M. C.; XAVIER, J. J. B. N.; BARRENTO, J. F.; FUKUDA, W. M. G. Aipim Manteiga: Cultivar de Macaxeira para o Amazonas. Comunicado Técnico 17. ISSN 1517-3887, Manaus-AM, Dezembro, 2003.

EMPRESA BRASILEIRA DE PESQUISA AGROPECUÁRIA. Cultura da mandioca: aspectos socioeconômicos, melhoramento genético, sistemas de cultivo, manejo de pragas e doenças e agroindústria. Brasília, DF: Embrapa, 2016. ISBN 978-85-7035-621-5. PDF 257 p.; il. color.: $15 \mathrm{~cm} \times 21 \mathrm{~cm}$. Disponível em: https://www.embrapa.br/amazonia-oriental/publicacoes. Acesso em: 15/01/2019.

EMPRESA BRASILEIRA DE PESQUISA AGROPECUÁRIA. Mandioca em números. Brasília, DF: Embrapa, 2017. Disponível em: https://www.embrapa.br/congresso-de-mandioca-2018/mandioca-em-numeros. Acesso em: $15 / 01 / 2019$.

EMPRESA BRASILEIRA DE PESQUISA AGROPECUÁRIA. Manual de métodos de análise de solo. Centro Nacional de Pesquisa de Solos. 2. ed. rev. atual. - Rio de Janeiro, 1997. 212 p.

EMPRESA BRASILEIRA DE PESQUISA AGROPECUÁRIA. Produção de Biomassa de Mandioca. Mandioca em foco. Embrapa Mandioca e Fruticultura Tropical. n. 34. Cruz das Almas, BA. dez. 2007.

EMPRESA BRASILEIRA DE PESQUISA AGROPECUÁRIA. SISTEMA DE CLASSIFICAÇÃO DE SOLOS. $2^{\mathrm{a}}$ edição. Embrapa Solos. Ministério da Agricultura, Pecuária e Abastecimento. Brasília, DF. 2006.

FERREIRA, D. F. Sisvar: a computer statistical analysis system. Ciência e Agrotecnologia (UFLA), v. 35, n.6, p. 1039-1042, 2011.

FERREIRA, G. D. G., OLIVEIRA, R. L., CARDOSO, E. d. C., MAGALHÃES, A. L. R. \& BRITO, E. L. 2008. Valor nutritivo de co-produtos da mandioca. Revista Brasileira de Saúde e Produção Animal, 8, 364-374.

FOOD AND AGRICULTURE ORGANIZATION OF UNITED STATES. FAOSTAT: Production Quantity of Cassava in 2017. Dec. 2018. Disponível em: http://www.fao.org/faostat/en/\#data/QC. Acesso em: 15/01/2019.

FUKUDA, W. M. G.; GUEVARA, C. L. Descritores morfológicos e agronômicos para a caracterização da mandioca (Manihot esculenta Crantz). Cruz das Almas: EMBRAPA- NPMF, 1998. 38p. (Embrapa-CNPMF. Documentos, 78).

KOTTEK, M.; GRIESER, J.; BECK, C.; RUDOLF, B.; RUBEL, F. World Map of the Köppen-Geiger climate classification updated. Meteorologische Zeitschrift, v. 15, n. 3, p. 259-263, 2006.

LENIS, J. I.; CALLE, F.; JARAMILlO, G.; PEREZ, J. C.; CEBALlOS, H.; COCK, J. H. Leaf retention and cassava productivity. Field Crops Research, v. 95, n. 2-3, p. 126-134, 2006.

LOZANO, J.C.; TORO, J.C. CASTRO, A.; BELLOTTI, A.C. 1977. Produção de material de plantio de mandioca. Cali, Colombia: Centro Internacional de Agricultura Tropical. 29p.

MIRANDA, L. N.; FIALHO, J. F.; MIRANDA, J. C. C.; GOMES, A. C. Manejo da calagem e da adubação fosfatada para a cultura da mandioca em solo de cerrado. Planaltina, DF: Embrapa Cerrados, 2005. (Embrapa Cerrados. Comunicado técnico, 118).

MODESTO, E. C.; SANTOS, G. T. dos; FILHO, P. S. V.; ZABOM, M. A.; VILELA, D.; JOBIM, C. C.; FARIA, K. P.; DETMANN, E. COMPOSIÇÃO QUÍMICA DAS FOLHAS DE CINCO CULTIVARES DE MANDIOCA (Manihot esculenta Crantz.) EM DIFERENTES ÉPOCAS DE COLHEITA. Departamento de Zootecnia da Universidade Estadual de Maringá, Maringá - PR, 1999.

OLIVEIRA, S. L. de; COELHO, E. F.; NOGUEIRA, C. C. P. Irrigação. In: Aspectos Socioeconomicos e Agronomicos da Mandioca. Editor: Luciano da Silva Souza...[et al.].-Cruz das Almas:Embrapa Mandioca e Fruticultura Tropical, 2006. P.291-300. 
PONTE, C. M. de A. Épocas de colheita de variedades de mandioca. 2008. 108f. Dissertação (Mestrado em Agronomia) - Universidade Estadual do Sudoeste da Bahia, Vitória da Conquista.

RIMOLDI, F.; VIDIGAL FILHO, P. S.; VIDIGAL, M. C. G.; CLEMENTE, E.; PEQUENO, M. G.; MIRANDA, L.; KVITSCHAL, M. V. Produtividade, composição química e tempo de cozimento de cultivares de mandioca de mesa coletadas no Estado do Paraná. Acta Sci Agron., Maringá, v. 28, n 1, p. 63-69, 2006.

SOCIEDADE BRASILEIRA DE CIÊNCIA DO SOLO. Fertilidade do solo. Viçosa-MG, 2007. 1017p. ISBN 978-85-8650408-2.

SOUZA, J. Folha de mandioca: alternativa alimentar. 2007. Apud SANTOS, E. F.; CARVALHO, F. S.; SILVA, J. C. G.; REZENDE, A. A.; MIYAJI, M. AGROINDÚSTRIA DA MANDIOCA - O CAMINHO PARA A SUSTENTABILIDADE ECONÔMICA DOS BENEFICIADORES DO BAIRRO CAMPINHOS EM VITÓRIA DA CONQUISTA - BA. In: 47º CONGRESSO SOBER. Sociedade Brasileira de Economia, Administração e Sociologia Rural: Desenvolvimento Rural e Sistemas Agroalimentares: Os Agronegócios no Contexto de Integração das Nações. 2009. Porto AlegreRS. Apresentação Oral-Agricultura Familiar e Ruralidade. Porto Alegre, RS. 2009.

SOUZA, L. S.; SILVA, J.; SOUZA, L. D. Recomendação de calagem e adubação para o cultivo de mandioca. Cruz das Almas: Embrapa Mandioca e Fruticultura, 2009. 6 p. (Embrapa Mandioca e Fruticultura. Comunicado técnico, 133).

TEZARA, W. et al. Effects of water deficit and its interaction with $\mathrm{CO}_{2}$ supply on the biochemistry and physiology of photosynthesis in sunflower. Journal of Experimental Botany, Oxford, v.53, n.375, p.1781-1791, 2002.

VALLE, T. L. Cruzamentos dialélicos em mandioca (Manihot esculenta Crantz). 1990. 180p. Tese (Doutorado em Agronomia) - Escola Superior de Agricultura "Luiz de Queiroz" da Universidade de São Paulo, Piracicaba.

VALLE, T. L.; ZATARIM, M.; MUHLEN, G. S.; GALERA, J. M. S. V.; FELTRAN, J. C. Variedades e diversidade genética de mandioca (Manihot esculenta Crantz) no Estado de Mato Grosso do Sul. In: XI CONGRESSO BRASILEIRO DE MANDIOCA, 2005, Campo Grande MS. Anais do XI Congresso Brasileiro de Mandioca. Campo Grande MS, 2005.

\section{(cc) EY}

Este trabalho está licenciado com uma Licença Creative Commons - Atribuição 4.0 Internacional. 